\title{
基于线粒体基因组数据的扁甲系总科间系统发育 关系分析
}

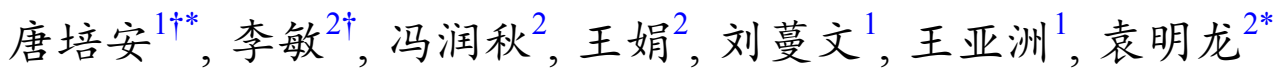

1. 南京财经大学食品科学与工程学院, 江苏省现代粮食流通与安全协同创新中心, 南京 210023 ;

2. 兰州大学草地农业生态系统国家重点实验室, 兰州大学农业农村部草牧业创新重点实验室, 兰州大学草地农业科技学院, 兰州 730020

$\dagger$ 同等贡献

*联系人, E-mail: tangpeian@163.com; yuanml@1zu.edu.cn

收稿日期：2018-06-14; 接受日期：2018-10-29; 网络版发表日期：2019-01-14

国家重点研发计划(批准号: 2016YFD0401004-4, 2017YFD0401003-5)、江苏省六大人才高峰项目(批准号: NY-057)、江苏省高校自然科学研 究面上项目(批准号: 17KJB210005)、国家自然科学基金(批准号: 31000828)和江苏高校优势学科建设工程资助

摘要 扁甲系是鞘翅目多食亚目中最大的一个类群, 具有重要的经济意义. 尽管扁甲系昆虫的单系性得到广泛支 持, 但7个总科间的系统进化关系仍存在不确定性. 为此, 本研究测定了锈赤扁谷盗和锯谷盗的线粒体基因组, 并 联合已测的 67 个扁甲系昆虫线粒体基因组, 构建了扁甲系昆虫总科间的系统发育关系, 并采用似然值检验对不同 的树拓扑结构进行了评价. 结果表明, 锈赤扁谷盗全线粒体基因组的大小为 $15497 \mathrm{bp}$, 编码 13 个蛋白质编码基 因、22个转运RNA基因和 2 个核糖体RNA基因。锯谷盗接近全长的线粒体基因长为 $13588 \mathrm{bp}$, 编码 34 个基因，缺 少 $\operatorname{trn} I, \operatorname{trn} Q$ 和 $\operatorname{trn} M$ 等 3 个转运 RNA基因. 锈赤扁谷盗和锯谷盗线粒体基因的基因含量、基因排序、碱基组成、密 码子使用等特征, 与大多数已测鞘翅目昆虫的线粒体基因组高度相似. 基于 3 个线粒体基因数据集及 3 种树构建方 法的系统发育分析结果表明，漂虫总科、叶甲总科、象甲总科、拟步甲总科和郭公虫总科等5个类群均为单系 性，而扁甲总科始终为并系。树拓扑结构检验结果表明，扁甲系总科间的系统发育关系为((郭公虫总科十漂虫总 科) $+($ 拟步甲总科 $+($ 扁甲总科(叶甲总科十象甲总科) $))$ ). 尽管线粒体数据集的构成及系统发育分析方法对树拓扑结 构具有重要影响, 但线粒体基因组数据在扁甲系昆虫系统与进化研究方面具有重要应用价值.

关键词 线粒体DNA, 甲虫, 系统发育分析, 最大似然法, 贝叶斯推断, 拓扑结构检验

扁甲系(Cucujiformia)是鞘翅目(Coleoptera)多食亚 目(Polyphaga)中最大的一个类群，目前全世界已报道 的种类超过 173000 种 $^{[1]}$. 扁甲系昆虫具有重要的经济 意义，既包括许多重大的农林牧业害虫，也包括众多 重要的天敌昆虫、传粉昆虫和药用昆虫. 目前, 扁甲
系昆虫通常分为6个总科，即郭公虫总科(Cleroidea)、 扁甲总科(Cucujoidea)、象甲总科(Curculionoidea)、 叶甲总科(Chrysomeloidea)、拟步甲总科(Tenebrionoidea)和筒蠹总科(Lymexyloidea $)^{[1 \sim 3]}$. 在过去的 20 多年 中，基于形态学特征及分子数据对扁甲系昆虫的系统

引用格式: 唐培安, 李敏, 冯润秋, 等. 基于线粒体基因组数据的扁甲系总科间系统发育关系分析. 中国科学: 生命科学, 2019, 49: 163-171 Tang P A, Li M, Feng R Q, et al. Phylogenetic relationships among superfamilies of Cucujiformia (Coleoptera: Polyphaga) inferred from mitogenomic data (in Chinese). Sci Sin Vitae, 2019, 49: 163-171, doi: 10.1360/N052018-00119 
进化关系进行了广泛探讨，但有关总科间的系统发育 关系还未完全解决 ${ }^{[1,2,45]}$. 特别是, 有关扁甲总科的单 系性及其系统发育地位最具争议性. Hunt等人 ${ }^{[2]}$ 基于 3 个基因( coxl $1,16 S$ 和 18S) 首次大规模分析了鞘翅目 1900 余种的系统发育关系, 发现扁甲总科是一并系群, 且支 持皮坚甲群(cerylonid group或cerylonid series)的单系 性；这一结论也被之后的形态学及分子系统发育研究 所支持 ${ }^{[6,7]}$. 目前，皮坚甲群已被正式命名为扁甲系的 一个新总科, 即慓虫总科(Coccinelloidea $)^{[1]}$. 然而, 㼼虫 总科与扁甲总科的系统发育关系、扁甲总科其余科的 单系性及科间的系统发育关系仍存在不确定性 ${ }^{[1,2,6]}$.

昆虫线粒体基因组是一个大约15 18 kb的环状双 链DNA分子，通常编码 37 个基因，即 13 个蛋白质编码 基因(PCGs)、22个转运RNA基因(tRNAs)和 2 个核糖 体RNA基因 $(r r n L \text { 和 } r r n S)^{[8,9]}$. 此外, 线粒体基因组通常 还有一个负责复制和转录的非编码区, 即控制区 ${ }^{[9]}$. 由 于线粒体基因组具有母系遗传、多拷贝、缺少遗传重 组以及进化速率快等特点, 已广泛应用于比较基因组 学、分子进化、系统发育重建、谱系地理学及种群遗 传学等研究领域 ${ }^{[8,10,11]}$. 在过去的 10 年中, 昆虫线粒体 基因组研究受到了高度关注，并由于高通量测序技术 的广泛应用而使得已测线粒体基因组的昆虫种类呈快 速增长的趋势. 目前, 线粒体基因组数据广泛用于探讨 鞘翅目昆虫不同分类阶元的系统进化关系，并高度支 持扁甲系的单系性 ${ }^{[4,5]}$. 然而，基于线粒体基因组数据 的扁甲系总科间的系统进化关系仍具有不确定性. 一 方面，一些类群仅有较少的物种进行了线粒体基因组 测序，如扁甲总科昆虫; 另一方面，不同的线粒体基因 数据集的构成、不同的系统发育分析方法对系统发育 的构建的结果也存在重要影响. 因此, 测序更多扁甲系 昆虫, 特别是扁甲总科昆虫的线粒体基因组, 并比较分 析不同系统发育分析方法在构建扁甲系系统进化关系 方面的价值和潜力, 显得非常必要.

本研究测定了锈赤扁谷盗(Cryptolestes ferrugi$n e u s$ )和锯谷盗(Oryzaephilus surinamensis)的线粒体基 因组, 进一步丰富了扁甲总科的线粒体基因组数量. 在 注释这 2 个新测线粒体基因组的基础上，联合 NCBI中 的 67 个扁甲系昆虫的线粒体基因组，基于 3 个线粒体 基因组数据集(P123，P123_2rRNA和AA), 采用 3 种方 法(RAxML，MrBayes和PhyloBayes)构建了扁甲系昆 虫总科间的系统发育关系，并采用似然值检验对不同
的树拓扑结构进行了评价.

\section{1 材料与方法}

\section{1 基因组DNA提取、PCR扩增及测序}

锈赤扁谷盗和锯谷盗成虫样本于 2016 年分别采集 于江苏苏州和湖南常德, 采集活体后, 浸泡在无水乙醇 中, 并保存于 $-80^{\circ} \mathrm{C}$. 采用DNA提取试剂盒(天根生物, 北京)提取单头昆虫的基因组总DNA. 采用昆虫通用引 物及新设计的特异引物扩增锈赤扁谷盗和锯谷盗的线 粒体基因组，引物序列及来源见网络版表1.PCR扩增 采用LA Taq酶(TaKaRa，日本)在 $25 \mu \mathrm{L}$ 体系中进行; 所 有PCR扩增片段，经电泳检测、纯化后送至上海生工 生物工程技术服务有限公司直接进行双向测序(测序 引物同PCR扩增); 对于测序失败或测序结果不理想的 片段, 克隆后送菌液进行双向测序.

\section{2 序列分析}

为保证序列的准确读取, 对测序结果进行人工校 正，保证碱基识别与原始测序峰图的一致性. 去除载 体及引物序列后，对每一PCR片段的双向测序结果进 行拼接，获得该目的片段的正确序列。锈赤扁谷盗和 锯谷盗的 13 个线粒体蛋白编码基因、2个rRNA基因及 22 个tRNA基因的注释，均采用已发表文献 ${ }^{[12,13]}$ 中的方 法进行. 蛋白质编码基因采用 NCBI的在线软件 ORF (open reading frame)进行鉴定; 蛋白编码基因确定后, 两个最大的非编码区分别作为核糖体大亚基基因 $(r r n L)$ 和小亚基基因 $(r r n S)$, 并进行Blast比对; 采用软件 tRNAscan-SE 1.21 $1^{[14]}$ 鉴定tRNA基因, 并预测其二级结 构. 采用Mega $6.06^{[15]}$ 分析碱基组成和密码子使用, 并 计算 $\mathrm{AT}-$ 偏斜 $=[\mathrm{A}-\mathrm{T}] /[\mathrm{A}+\mathrm{T}]$ 和 $\mathrm{GC}-$ 偏斜 $=[\mathrm{G}-\mathrm{C}] /[\mathrm{G}$ $+\mathrm{C}]^{[16]}$.

\section{3 系统发育分析}

采用本研究新测的锈赤扁谷盗和锯谷盗线粒体基 因组，联合GenBank中已有的67种扁甲系昆虫线粒体 基因组进行系统发育分析，并以金龟甲系(Scarabaeiformia)的 2 个物种(Lucanus mazama和Rhopaea magnicornis)作为外群(网络版表2). 采用TranslatorX online server ${ }^{[17]}$ 的MAFFT对13个蛋白编码基因分别进行序列 
比对, 并采用GBlocks去除空位及模糊位点, 将比对好 的单个蛋白质编码基因联合在一起获得线粒体基因数 据集. 两个rRNA基因的序列比对，采用已发表文献 ${ }^{[4]}$ 中的方法进行. DAMBE分析结果表明, 除rrnS 在不对 称树 (asymmetrical tree)的情况下达到饱和外, 其余数 据集均未达到显著饱和(网络版表3). 为了明确线粒体 数据集的构成差异对系统发育结果的影响, 构建了 3 个 线粒体数据集: 即P123(核苷酸序列, 包括 13 个蛋白质 编码基因的 3 个密码子位点)、P123_2rRNA(核苷酸序 列，P123加上 2 个核糖体rRNA基因)和 $\mathrm{AA}(\mathrm{P} 123$ 对应的 氨基酸序列). 对这 3 个数据集, 分别采用PartitionFinder 1.1.1 ${ }^{[18]}$ 选择序列的最佳分区及进化模型，并将结果用 于后续系统发育分析(网络版表4).

对P123，P123_2rRNA和AA等3个数据集, 分别采 用RAXML(ML), MrBayes(BI)和PhyloBayes等3种方法 构建扁甲系昆虫的系统进化关系. 所有系统发育分析, 均在CIPRES Science Gateway $3.3^{[19]}$ 在线平台上完成. ML分析用RAxML-HPC2 on XSEDE 8.0.24 $4^{[20]}$ 进行, 采 用GTRGAMMAI模型, 支的可靠性采用 1000 次的bootstrap进行评估. 贝叶斯BI分析采用MrBayes 3.2.2 $2^{[21]}$ 进 行, 4条独立的马尔可夫链(Markov chains), 即3 条热链 (hot chain)和 1 条冷链(cold chain) 同时运行 $1 \times 10^{8}$ 代 (generation). 每运行1000带取样一次, 当ESS(estimated sample size) $>100$ 且 PSRF(potential scale reduction factor)接近 1.0 时即认为两个分析过程趋于稳定状态. 舍 弃 $25 \%$ 的老化样本, 剩余样本用来构建 $50 \%$ 一致树, 并 计算贝叶斯后验概率. PhyloBayes分析采用PhyloBayes MPI $1.5 \mathrm{a}$ 在贝叶斯位点异质模型(site-heterogeneous model)下进行; 运行时, 移除不变位点, 2 条独立的链 起始于一个随机树，并设置为CAT-poisson模型；其余 参数为CIPRES Science Gateway平台上PhyloBayes软 件的默认设置.

由于不同的数据集及树构建方法获得的扁甲系总 科间的系统发育关系存在不一致性，因此为了明确哪 种系统发育树值得信任，我们采用IQ-TREE web server(http://iqtree.cibiv.univie.ac.at/)进行树拓扑结构检 验 ${ }^{[22]}$. 对P123, P123_2rRNA和AA等3个数据集, 分别采 用 SH(Shimodaira-Hasegawa test) ${ }^{[23]}$, WKH(weighted Kishino-Hasegawa test) ${ }^{[24]}$, WSH(weighted SH test) ${ }^{[23]}$ 和AU(approximately unbiased test) ${ }^{[25]}$ 等4种方法进行统 计分析, 并设置1000次重复(replicates).

\section{2 结果与分析}

\section{1 锈赤扁谷盗和锯谷盗线粒体基因组的基本 特征}

通过PCR扩增、测序，我们获得了锈赤扁谷盗完 整的线粒体基因组(GenBank登录号：MH475917)以及 接近全长的锯谷盗线粒体基因组(GenBank登录号: MH475916). 锈赤扁谷盗线粒体基因组是一个典型的 闭合环状双链DNA分子, 大小为 $15497 \mathrm{bp}$ (网络版表5 和网络版表6), 编码 37 个线粒体基因, 即 13 个PCGs, 22 个tRNAs和 2 个rRNAs，且基因排序与节肢动物线粒体 基因的原始排序完全一致 ${ }^{[8,9]}$. 锯谷盗线粒体基因组大 小为 $13588 \mathrm{bp}$, 编码 34 个基因, 缺少 $\operatorname{trnI}, \operatorname{trnQ}$ 和 $\operatorname{trnM}$ 等 3 个位于控制区附近的tRNAs(网络版表5和网络版表6).

锈赤扁谷盗和锯谷盗线粒体基因组结构较为紧 凑，除线粒体控制区外，仅有几个小的非编码区，即基 因间隔区. 其中，最大的基因间隔区均位于 $t r n S 2$ 和 $n a d 1$ 之间, 大小分别为 18 和 $28 \mathrm{bp}$ (网络版表 5 和网络版 表6). 研究表明, 该基因间隔区所在的位置及序列在不 同昆虫中具有保守性 ${ }^{[4,26,27]}$, 且包含可与线粒体转录因 子DmTTF结合的位点 ${ }^{[28]}$. 与其他已测目昆虫相似, 锈 赤扁谷盗和锯谷盗线粒体基因组的碱基组成明显偏向 于 $\mathrm{A}+\mathrm{T}: \mathrm{J}-\mathrm{c}$ 链的 $\mathrm{A} \%+\mathrm{T} \%$ 分别为 $77.0 \%$ 和 $72.1 \%, 13$ 个 $\mathrm{PCGs}$ 的 $\mathrm{A} \%+\mathrm{T} \%$ 含量分别为 $75.4 \%$ 和 $70.9 \%$, tRNAs的 $\mathrm{A} \%+\mathrm{T} \%$ 分别为 $77.5 \%$ 和 $78.1 \%, r r n L$ 的 $\mathrm{A} \%+\mathrm{T} \%$ 分别为 $82.0 \%$ 和 $76.4 \%$. 锈赤扁谷盗和锯谷盗 13 个 PCGs的 3 个 核苷酸位点, 其 $\mathrm{A} \%+\mathrm{T} \%$ 有明显差异, 第3位点的 $\mathrm{A} \%+\mathrm{T}$ $\%$ 均明显高于前两个位点(表1). 两个线粒体基因组J链的 AT-偏斜均为正值 $(0.101)$, 而 $\mathrm{GC}-$ 偏斜为负值 $(-$ 0.181 ), 这与大多数昆虫线粒体基因组核苷酸偏斜的 规律一致 ${ }^{[29]}$.

锈赤扁谷盗和锯谷盗线粒体基因组高的 $\mathrm{A}+\mathrm{T}$ 含量 及不同链上核苷酸的偏斜性，也反映在蛋白质编码基 因的密码子使用上(网络版表7). 相对同义密码子使用 频率(relative synonymous codon usage, RSCU)分析表 明, 以 $\mathrm{A}$ 或 $\mathrm{T}$ 结尾的同义密码子出现的次数远大于其他 同义密码子出现的次数, 即AT含量丰富的密码子被频 繁使用(网络版表7). 锯谷盗线粒体基因组使用了无脊 椎动物的 62 种线粒体密码子，而锈赤扁谷盗线粒体基 因组没有使用UCG密码子. 6 个完全由A和T组成的密 码子(TTT, TTA, ATT, ATA, TAT和AAT)在锈赤扁谷盗 
唐培安等: 基于线粒体基因组数据的扁甲系总科间系统发育关系分析

表 1 锈赤扁谷盗和锯谷盗线粒体基因组的碱基组成

Table 1 Base composition of mitochondrial genomes of Cryptolestes ferrugineus and Oryzaephilus surinamensis

\begin{tabular}{|c|c|c|c|c|c|c|}
\hline & \multicolumn{3}{|c|}{ 锈赤扁谷盗 } & \multicolumn{3}{|c|}{ 锯谷盗 } \\
\hline & $\mathrm{A}+\mathrm{T}(\%)$ & AT-skew & GC-skew & $\mathrm{A}+\mathrm{T}(\%)$ & AT-skew & GC-skew \\
\hline 全线粒体基因组 & 77.0 & 0.014 & -0.200 & 72.1 & 0.136 & -0.325 \\
\hline 蛋白质编码基因 & 75.4 & -0.168 & -0.012 & 70.9 & -0.116 & -0.076 \\
\hline 蛋白质编码基因第 1 位点 & 69.3 & -0.037 & 0.167 & 66.6 & 0.019 & 0.116 \\
\hline 蛋白质编码基因第2位点 & 68.5 & -0.393 & -0.120 & 66.7 & -0.388 & -0.150 \\
\hline 蛋白质编码基因第3位点 & 88.4 & -0.098 & -0.191 & 79.5 & -0.001 & -0.270 \\
\hline 转运RNA基因 & 77.5 & 0.032 & 0.108 & 78.1 & 0.033 & 0.111 \\
\hline 核糖体16S基因 & 82.0 & -0.014 & 0.365 & 76.4 & -0.174 & 0.411 \\
\hline 核糖体12S基因 & 80.1 & 0.050 & 0.290 & & & \\
\hline 线粒体控制区 & 85.6 & -0.051 & -0.175 & & & \\
\hline
\end{tabular}

和锯谷盗线粒体基因组中的使用频率均最高, 它们的 含量占分别占 $45 \%$ 和 $35 \%$. 锈赤扁谷盗和锯谷盗线粒 体基因组的这种密码使用模式, 与已测其他扁甲系昆 虫的高度相似 ${ }^{[4]}$.

除nad1外，锈赤扁谷盗其余 12 个蛋白质编码基因 的起始密码子均为ATN, 而锯谷盗的 nad 1 和 $\operatorname{cox} 1$ 没有 以典型的ATN作为起始密码子. 锈赤扁谷盗和锯谷盗 的大多数蛋白质编码基因使用不完全的终止密码子 $\mathrm{T}$ 和 TA. 不完全的终止密码子在昆虫线粒体基因组中普 遍存在，推测在转录过程中通过添加 $\mathrm{A}$ 可以将这些不 完整的终止密码子补全而并不影响翻译 ${ }^{[30,31]}$. 除trnS1 外, 锈赤扁谷盗和锯谷盗的其余线粒体tRNAs均具有 经典的三叶草结构，包括 $\mathrm{T} \psi \mathrm{C}$ 臂、氨基酸接受臂、反 密码子臂和双氢尿嘧啶(dihydrouridine, DHU)臂.

\section{2 系统发育分析}

基于 3 个数据集(P123，P123_2rRNA 和AA)和 3 种 系统发育分析方法(RAxML, MrBayes和PhyloBayes), 总共获得了9个系统发育树(图1, 网络版图1 网络版图 8). 尽管这9个系统发育树的拓扑结构不完全一致，但 均不同程度地支持慓虫总科、叶甲总科、象甲总科和 拟步甲总科等 4 个总科的单系性(表 1$)$. 除 2 个数据集 (P123和P123_2rRNA)的ML树外，其余7个系统发育分 析均不同程度支持郭公虫总科的单系性. 相反, 所有的 系统发育分析均不支持扁甲总科的单系性，这与基于 形态学及分子数据的已有研究结果一致 ${ }^{[1,2,4 \sim 7,32,33]}$. 扁 甲总科是多食亚目扁甲系中种类多、系统分类仍有争
议的一个类群, 目前包括 25 个科 ${ }^{[1,2,6,7]}$. 本研究测定的 锈赤扁谷盗和锯谷盗分别代表了扁谷盗科(Laemophloeidae)和锯谷盗科(Silvanidae), 将扁甲总科中已测线 粒体基因组的科数从 8 个增加至 10 个. 尽管物种数及 科数的增加并未解决扁甲总科的并系性问题, 但本研 究的结果支持扁甲总科与象甲科和叶甲科构成一单系 群(图1, 网络版图1 网络版图8).

三种系统发育树构建方法中, ML树的支持率相对 较低; 例如, ML树对叶甲总科单系性的支持率均小于 70 , 而BI树和PhyloBayes树的支持率均大于 0.92 (表1). 此外, 某些被贝叶斯系统树支持的系统发育关系, 并 不被 ML树支持. 例如, 基于 2 个数据集(P123和 P123_2rRNA)的BI树和PhyloBayes树均支持拟步甲总 科与扁甲总科、叶甲总科和象甲总科等 3 个总科构成 姊妹群关系, 但ML树并不支持这一关系(表1). 这表明, 尽管采用RAxML的最大似然法在系统发育分析中广 泛应用，但该软件在构建某些类群的系统发育关系方 面有时表现欠佳, 这与其他已有研究结果一致 ${ }^{[4,13,34,35]}$.

综合比较 9 个系统发育树发现, 核苷酸数据较氨基 酸数据表现出更好的系统发育信息(表2, 图1, 网络版 图1 网络版图 8). 例如, 基于 2 个数据集(P123和 P123_2rRNA)的BI树均支持叶甲总科与象甲总科为姊 妹群关系, 但氨基酸数据集的 $\mathrm{BI}$ 树并不支持这一关系. 尽管 $r r n S$ 表现出了一定程度的序列饱和度, 但与 $\mathrm{P} 123$ 相比，包含 2 个rRNA基因的数据集(P123_2rRNA)具有 更高的系统发育分辨率，例如，基于P123_2rRNA和 P123的ML树，仅前者支持叶甲总科与象甲总科为姊 


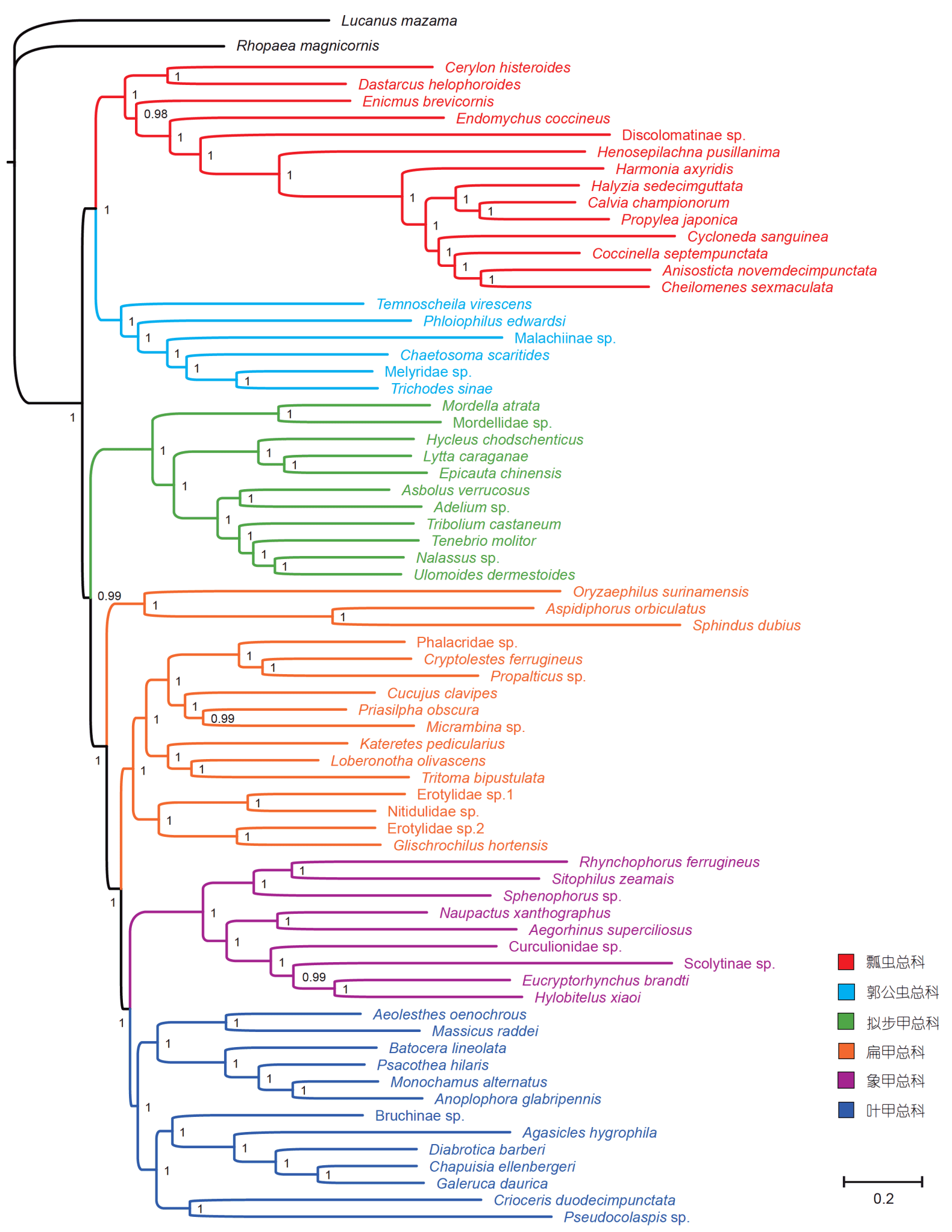

图 1 基于P123_2rRNA的贝叶斯(MrBayes)系统发育树

Figure 1 Bayesian phylogenetic tree based on the P123_2rRNA dataset 
表 2 不同数据集、不同方法获得的扁甲系总科间的系统发育结果 ${ }^{a)}$

Table 2 Phylogenetic relationships among superfamilies with Cucujiformia based on different datasets and analytical methods

\begin{tabular}{|c|c|c|c|c|c|c|c|c|c|}
\hline \multirow{2}{*}{ 系统发育关系 } & \multicolumn{3}{|c|}{ P123 } & \multicolumn{3}{|c|}{ P123_2rRNA } & \multicolumn{3}{|c|}{ AA } \\
\hline & BI & ML & PhyloBayes & BI & ML & PhyloBayes & BI & ML & PhyloBayes \\
\hline 郭公虫总科是单系群 & 0.68 & - & 1 & 1 & - & 1 & 1 & 99 & 1 \\
\hline 慓虫总科是单系群 & 1 & 82 & 1 & 1 & 84 & 1 & 1 & 96 & 1 \\
\hline 叶甲总科是单系群 & 1 & 46 & 0.99 & 1 & 67 & 0.99 & 0.92 & 55 & 0.99 \\
\hline 扁甲总科是单系群 & - & - & - & - & - & - & - & - & - \\
\hline 象甲总科是单系群 & 1 & 100 & 1 & 1 & 100 & 1 & 1 & 100 & 1 \\
\hline 拟步甲总科是单系群 & 1 & 100 & 1 & 1 & 100 & 1 & 1 & 100 & 1 \\
\hline $\mathrm{CLE}+\mathrm{COC}$ & 0.68 & - & - & 1 & - & - & - & - & - \\
\hline (CLE, COC)+(TEN, CUC, CHR, CUR) & 1 & - & - & 1 & - & - & - & - & - \\
\hline TEN+(CUC, CHR, CUR) & 0.68 & - & 0.87 & 0.99 & - & 0.83 & - & - & 0.98 \\
\hline $\mathrm{CUC}+(\mathrm{CHR}, \mathrm{CUR})$ & - & - & - & - & - & - & - & - & - \\
\hline CLE+(COC, TEN, CUC, CHR, CUR) & - & - & 1 & - & - & 1 & 1 & 100 & 1 \\
\hline $\mathrm{COC}+(\mathrm{TEN}, \mathrm{CUC}, \mathrm{CHR}, \mathrm{CUR})$ & - & - & 0.83 & - & - & 0.59 & - & - & 0.89 \\
\hline $\mathrm{CHR}+\mathrm{CUR}$ & 0.98 & - & 0.99 & 1 & 44 & 0.99 & - & - & 0.87 \\
\hline $\mathrm{TEN}+\mathrm{COC}$ & - & - & - & - & - & - & 0.78 & 41 & - \\
\hline$(\mathrm{TEN}, \mathrm{COC})+(\mathrm{CUC}, \mathrm{CHR}, \mathrm{CUR})$ & - & - & - & - & - & - & 1 & 78 & - \\
\hline
\end{tabular}

a) CLE, 郭公虫总科; COC, 漂虫总科; TEN, 拟步甲总科; CUC, 扁甲总科; CHR, 叶甲总科; CUR, 象甲总科

妹群. 这表明，包含RNA的线粒体基因组数据更有助 于解决扁甲系总科间的系统发育关系，这与作者前面 的研究结果一致 ${ }^{[4]}$.

\section{3 树拓扑结构检验}

按照扁甲总科间的系统发育关系, 本研究的9个系 统发育树共有 5 种不同的树拓扑结构(表3)。树拓扑结 构检验结果表明，基于 3 个数据集的树拓扑结构检验, 获得了高度一致的结果，均支持P123和P123 2rRNA 两个数据集BI树总科间的系统发育关系，即((郭公虫 总科+㼼虫总科)，(拟步甲总科 + (扁甲总科(叶甲总科 + 象甲总科)) ))(表 3, 图1). 叶甲总科与象甲总科互为姊 妹群，这一关系在前面的分子系统研究中得到了广泛 支持 ${ }^{[2,4 \sim 6,32]}$. 与之前基于线粒体基因组数据的研究结 果相同 ${ }^{[4,5]}$ ，原扁甲总科中的皮坚甲群，即最新命名的 漂虫总科在所有系统发育树中均为单系群，但其系统 位置在不同的系统发育树中不完全一致。系统发育结 果表明, 漂虫总科的系统位置有3种情况(表3): ( i ) 拓 扑结构检验支持度最高的是瓢虫总科与郭公虫总科互 为姊妹群, 这一关系也与之前的研究结果一致 ${ }^{[2,4]}$; (ii)
漂虫总科位于除郭公虫总科外其余 4 个总科的基部, 拓 扑结构检验支持度较低，但前面的一些研究也发现该 系统关系 ${ }^{[32]}$; (iii) 慓虫总科与拟步甲总科为姊妹群, 尽管该结果与之前的研究一致 ${ }^{[5]}$, 但拓扑结构检验完 全不支持这一关系. 基于相同数据集的 $\mathrm{SH}$ 检验结果表 明, 漂虫总科前两种系统位置的概率较为接近, 表明漂 虫总科的系统位置还需进一步探讨.

不同的研究因在数据集构成、分析方法及物种数 量等方面存在差异，获得的扁甲总科科间的系统发育 关系差异很大. Hunt等人 ${ }^{[2]}$ 基于 3 个基因 $(\operatorname{cox} 1 ， 16 S$ 和 $18 S$ )的系统发育分析发现，小草甲科(Sphindidae)与皮 扁甲科(Phloeostichidae)聚在一起，且与象甲总科为姊 妹群关系．尽管扁甲科(Cucujidae)很可能是锯谷盗科

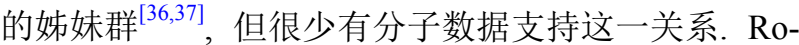
bertson等人 ${ }^{[1]}$ 基于 8 个基因的系统发育分析表明，扁甲 科与锯谷盗科为姊妹群关系，且两者与扁谷盗科聚在 一起. 本研究结果表明(图1), 扁甲总科分为两个单系 群：小萆甲科和锯谷盗科聚在一起，位于扁甲总科、 象甲总科和叶甲总科的最基部; 包括扁甲科和扁谷盗 科在内的其余 8 个科构成了另一个单系群，与象甲总 
表 3 系统发育树拓扑结构检验结果 ${ }^{\text {a) }}$

Table 3 Results of topological tests for five phylogenetic trees

\begin{tabular}{|c|c|c|c|c|c|c|}
\hline 数据集 & 拓扑结构 & -Ln likelihood & $\mathrm{SH}$ & WKH & WSH & $\mathrm{AU}$ \\
\hline \multirow[t]{5}{*}{ P123 } & 1. (outgroup, (느, COC, TEN, $\underline{\text { CUC }}$, CHR, CUR)) & -424561.9 & 0 & 0 & 0 & 0.01 \\
\hline & 2. (outgroup, ((CLE, COC), (TEN, ( $\underline{\mathrm{CUC}},(\mathrm{CHR}, \mathrm{CUR})))))$ & -423109.3 & 0.86 & 0.79 & 0.98 & 0.77 \\
\hline & 3. (outgroup, (CLE, (COC, (TEN, (CUC, (CHR, CUR)))))) & -423114.5 & 0.82 & 0.20 & 0.61 & 0.22 \\
\hline & 4. (outgroup, ( $\underline{\mathrm{CLE}}, \mathrm{COC}, \mathrm{TEN},(\underline{\mathrm{CUC}},(\mathrm{CHR}, \mathrm{CUR}))))$ & -423473.8 & 0 & 0 & 0 & 0 \\
\hline & 5. (outgroup, (CLE, ((COC, TEN), (CUC, CHR, CUR)))) & -423196.2 & 0.25 & 0 & 0 & 0.01 \\
\hline \multirow[t]{5}{*}{ P123_2rRNA } & 1. (outgroup, (CLE, COC, TEN, CUC, CHR, CUR)) & -460963.0 & 0 & 0 & 0 & 0 \\
\hline & 2. (outgroup, ((CLE, COC), (TEN, (CUC, $($ CHR, CUR $))))$ ) & -459379.3 & 0.87 & 0.82 & 0.99 & 0.83 \\
\hline & 3. (outgroup, (CLE, (COC, (TEN, (CUC, (CHR, CUR)))))) & -459386.1 & 0.82 & 0.17 & 0.52 & 0.16 \\
\hline & 4. (outgroup, ( $\underline{\mathrm{CLE}}, \mathrm{COC}, \mathrm{TEN},(\underline{\mathrm{CUC}},(\mathrm{CHR}, \mathrm{CUR}))))$ & -459792.6 & 0 & 0 & 0 & 0.01 \\
\hline & 5. (outgroup, (CLE, ((COC, TEN), (CUC, CHR, CUR)))) & -459481.1 & 0.20 & 0 & 0 & 0.01 \\
\hline \multirow[t]{5}{*}{ Amino acid } & 1. (outgroup, ( $\underline{\mathrm{CLE}}, \mathrm{COC}, \mathrm{TEN}, \underline{\mathrm{CUC}}, \mathrm{CHR}, \mathrm{CUR})$ ) & -212140.1 & 0 & 0 & 0 & 0.01 \\
\hline & 2. (outgroup, ((CLE, COC), (TEN, ( $\underline{\mathrm{CUC}},(\mathrm{CHR}, \mathrm{CUR})))))$ & -210383.3 & 0.87 & 0.92 & 0.99 & 0.93 \\
\hline & 3. (outgroup, (CLE, (COC, (TEN, $(\underline{\mathrm{CUC}},(\mathrm{CHR}, \mathrm{CUR}))))))$ & -210395.9 & 0.73 & 0.07 & 0.28 & 0.06 \\
\hline & 4. (outgroup, ( $\underline{\mathrm{CLE}}, \mathrm{COC}, \mathrm{TEN},(\underline{\mathrm{CUC}},(\mathrm{CHR}, \mathrm{CUR}))))$ & -210811.9 & 0 & 0 & 0 & 0 \\
\hline & 5. (outgroup, (CLE, ((COC, TEN), (CUC, CHR, CUR)))) & -210479.6 & 0.25 & 0 & 0 & 0.01 \\
\hline
\end{tabular}

a) 下划线表示非单系群. CLE, 郭公虫总科; COC, 漂虫总科; TEN, 拟步甲总科; CUC, 扁甲总科; CHR, 叶甲总科; CUR, 象甲总科.

科和叶甲总科为姊妹群关系。扁谷盗科与展扁甲科 (Propalticidae)为姊妹群关系，这与前面基于形态学及 分子数据的分析一致 ${ }^{[1,7]}$. 考虑到本研究仅包括了扁甲 总科 25 个科中的 10 个科，对其余 15 个科进行线粒体基 因组测序, 并联合核基因和形态学数据进行综合分析, 可为扁甲总科内部类群的系统进化提供新的认识.

\section{3 结论}

本研究测定的锈赤扁谷盗和锯谷盗线粒体基因 组, 基因含量、基因排序、碱基组成、密码子使用模
式及tRNA二级结构等与大部分已测鞘翅目昆虫高度 相似. 基于 3 个线粒体基因数据集及 3 种树构建方法的 系统发育分析结果表明, 漂虫总科、叶甲总科、象甲 总科、拟步甲总科和郭公虫总科等 5 个类群均为单系 性，而扁甲总科始终为并系，树拓扑结构检验结果表 明，扁甲系总科间的系统发育关系为((郭公虫总科 + 瓢虫总科), (拟步甲总科 + (扁甲总科(叶甲总科 + 象 甲总科()))). 尽管线粒体数据集的构成及系统发育分 析方法对树拓扑结构具有重要影响, 但线粒体基因组 数据在扁甲系昆虫系统与进化研究方面具有重要应 用价值.

\section{参考文献}

1 Robertson J A, Ślipiński A, Moulton M, et al. Phylogeny and classification of Cucujoidea and the recognition of a new superfamily Coccinelloidea (Coleoptera: Cucujiformia). Syst Entomol, 2015, 40: 745-778

2 Hunt T, Bergsten J, Levkanicova Z, et al. A comprehensive phylogeny of beetles reveals the evolutionary origins of a superradiation. Science, 2007, 318: 1913-1916

3 Bouchard P, Bousquet Y, Davies A E, et al. Family-group names in Coleoptera (Insecta). Zookeys, 2011, 88: 1-972

4 Yuan M L, Zhang Q L, Zhang L, et al. High-level phylogeny of the Coleoptera inferred with mitochondrial genome sequences. Mol Phylogenets Evol, 2016, 104: 99-111 
5 Timmermans M J T N, Barton C, Haran J, et al. Family-level sampling of mitochondrial genomes in coleoptera: compositional heterogeneity and phylogenetics. Genome Biol Evol, 2016, 8: 161-175

6 Bocak L, Barton C, Crampton-platt A, et al. Building the Coleoptera tree-of-life for $>8000$ species: composition of public DNA data and fit with Linnaean classification. Syst Entomol, 2014, 39: 97-110

7 Lawrence J F, Ślipiński A, Seago A E, et al. Phylogeny of the Coleoptera based on morphological characters of adults and larvae. Ann Zool, 2011, 61: 1-217

8 Cameron S L. Insect mitochondrial genomics: implications for evolution and phylogeny. Annu Rev Entomol, 2014, 59: 95-117

9 Boore J L. Animal mitochondrial genomes. Nucl Acids Res, 1999, 27: 1767-1780

10 Simon C, Buckley T R, Frati F, et al. Incorporating molecular evolution into phylogenetic analysis, and a new compilation of conserved polymerase chain reaction primers for animal mitochondrial DNA. Annu Rev Ecol Evol Syst, 2006, 37: 545-579

11 Wang I J. Recognizing the temporal distinctions between landscape genetics and phylogeography. Mol Ecol, 2010, 19: 2605-2608

12 Yuan M L, Zhang Q L, Guo Z L, et al. Comparative mitogenomic analysis of the superfamily Pentatomoidea (Insecta: Hemiptera: Heteroptera) and phylogenetic implications. BMC Genom, 2015, 16: 460

13 Yuan M L, Zhang Q L, Guo Z L, et al. The complete mitochondrial genome of Corizus tetraspilus (Hemiptera: Rhopalidae) and phylogenetic analysis of Pentatomomorpha. PLoS ONE, 2015, 10: e0129003

14 Lowe T M, Eddy S R. tRNAscan-SE: a program for improved detection of transfer RNA genes in genomic sequence. Nucl Acids Res, 1997, 25: 955-964

15 Tamura K, Stecher G, Peterson D, et al. MEGA6: molecular evolutionary genetics analysis version 6.0. Mol Biol Evol, 2013, 30: 2725-2729

16 Perna N T, Kocher T D. Patterns of nucleotide composition at fourfold degenerate sites of animal mitochondrial genomes. J Mol Evol, 1995, 41: $353-358$

17 Abascal F, Zardoya R, Telford M J. TranslatorX: multiple alignment of nucleotide sequences guided by amino acid translations. Nucl Acids Res, 2010, 38: W7-W13

18 Lanfear R, Calcott B, Ho S Y W, et al. Partitionfinder: combined selection of partitioning schemes and substitution models for phylogenetic analyses. Mol Biol Evol, 2012, 29: 1695-1701

19 Miller MA, Pfeiffer W, Schwartz T. Creating the CIPRES Science Gateway for inference of large phylogenetic trees. New Orleans: Gateway Computing Environments Workshop (GCE), 2010. 1-8

20 Stamatakis A. RAxML version 8: a tool for phylogenetic analysis and post-analysis of large phylogenies. Bioinformatics, 2014, 30: 1312-1313

21 Ronquist F, Teslenko M, van der Mark P, et al. MrBayes 3.2: efficient Bayesian phylogenetic inference and model choice across a large model space. Syst Biol, 2012, 61: 539-542

22 Trifinopoulos J, Nguyen L T, von Haeseler A, et al. W-IQ-TREE: a fast online phylogenetic tool for maximum likelihood analysis. Nucl Acids Res, 2016, 44: W232-W235

23 Shimodaira H, Hasegawa M. Multiple comparisons of log-likelihoods with applications to phylogenetic inference. Mol Biol Evol, 1999, 16: $1114-1116$

24 Kishino H, Hasegawa M. Evaluation of the maximum likelihood estimate of the evolutionary tree topologies from DNA sequence data, and the branching order in hominoidea. J Mol Evol, 1989, 29: 170-179

25 Shimodaira H. An approximately unbiased test of phylogenetic tree selection. Syst Biol, 2002, 51: 492-508

26 Cameron S L, Whiting M F. The complete mitochondrial genome of the tobacco hornworm, Manduca sexta, (Insecta: Lepidoptera: Sphingidae), and an examination of mitochondrial gene variability within butterflies and moths. Gene, 2008, 408: 112-123

27 Beckenbach A T. Mitochondrial genome sequences of Nematocera (lower Diptera): evidence of rearrangement following a complete genome duplication in a winter crane fly. Genom Biol Evol, 2012, 4: 89-101

28 Roberti M, Polosa P L, Bruni F, et al. DmTTF, a novel mitochondrial transcription termination factor that recognises two sequences of Drosophila melanogaster mitochondrial DNA. Nucl Acids Res, 2003, 31: 1597-1604

29 Hassanin A, Leger N, Deutsch J. Evidence for multiple reversals of asymmetric mutational constraints during the evolution of the mitochondrial genome of Metazoa, and consequences for phylogenetic inferences. Syst Biol, 2005, 54: 277-298

30 Ojala D, Montoya J, Attardi G. tRNA punctuation model of RNA processing in human mitochondria. Nature, 1981, 290: 470-474

31 Lavrov D V, Boore J L, Brown W M. Complete mtDNA sequences of two millipedes suggest a new model for mitochondrial gene 
rearrangements: duplication and nonrandom loss. Mol Biol Evol, 2002, 19: 163-169

32 Crampton-Platt A, Timmermans M J T N, Gimmel M L, et al. Soup to tree: the phylogeny of beetles inferred by mitochondrial metagenomics of a Bornean rainforest sample. Mol Biol Evol, 2015, 32: 2302-2316

33 Marvaldi A E, Duckett C N, Kjer K M, et al. Structural alignment of $18 \mathrm{~S}$ and $28 \mathrm{~S}$ rDNA sequences provides insights into phylogeny of Phytophaga (Coleoptera: Curculionoidea and Chrysomeloidea). Zool Script, 2009, 38: 63-77

34 Wei S J, Li Q, van Achterberg K, et al. Two mitochondrial genomes from the families Bethylidae and Mutillidae: independent rearrangement of protein-coding genes and higher-level phylogeny of the Hymenoptera. Mol Phylogenet Evol, 2014, 77: 1-10

35 Mao M, Gibson T, Dowton M. Evolutionary dynamics of the mitochondrial genome in the Evaniomorpha (Hymenoptera) - a group with an intermediate rate of gene rearrangement. Genom Biol Evol, 2014, 6: 1862-1874

36 Leschen R A B, Lawrence J F, Ślipiński S A. Classification of basal Cucujoidea (Coleoptera:Polyphaga): cladistic analysis, keys and review of new families. Invert Syst, 2005, 19: 17-73

37 Mcelrath T C, Robertson J A, Thomas M C, et al. A molecular phylogenetic study of Cucujidae s.l. (Coleoptera: Cucujoidea). Syst Entomol, 2015, 40: 705-718

\title{
Phylogenetic relationships among superfamilies of Cucujiformia (Coleoptera: Polyphaga) inferred from mitogenomic data
}

\author{
TANG PeiAn ${ }^{1}$, LI Min ${ }^{2}$, FENG RunQiu ${ }^{2}$, WANG Juan ${ }^{2}$, LIU ManWen ${ }^{1}$, WANG YaZhou ${ }^{1}$ \\ \& YUAN MingLong ${ }^{2}$ \\ 1 Collaborative Innovation Center for Modern Grain Circulation and Safety, College of Food Science and Engineering, \\ Nanjing University of Finance and Economics, Nanjing 210023, China; \\ 2 State Key Laboratory of Grassland Agro-ecosystems, Lanzhou University; Key Laboratory of Grassland Livestock Industry Innovation, \\ Ministry of Agriculture and Rural Affairs, Lanzhou University; College of Pastoral Agriculture Science and Technology, Lanzhou University; \\ Lanzhou 730020, China
}

Cucujiformia is the largest group in the Polyphaga of the Coleoptera, most of which are of economic importance. Although the monophyly of Cucujiformia has been widely supported, the phylogenetic relationships among seven superfamilies within Cucujiformia remain unresolved. In this study, mitochondrial genomes (mitogenomes) of Cryptolestes ferrugineus and Oryzaephilus surinamensis were sequenced. Combined with other 67 sequenced coleopteran mitogenomes, we reconstructed phylogenetic relationships among superfamilies of Cucujiformia and evaluated tree topologies by likelihood value tests. The results showed that the complete mitogenome of C. ferrugineus was $15497 \mathrm{bp}$ long and encoded 13 protein-coding genes, 22 transfer RNA genes (tRNAs) and two ribosomal genes. The near complete mitogenome of $O$. surinamensis was $13588 \mathrm{bp}$ long and encoded 34 mitochondrial genes, lacking three tRNAs (trnI, $\operatorname{trn} Q$ and $\operatorname{trn} M$ ). General features of the two newly sequenced mitogenomes (gene content, gene arrangement, base composition and codon usage) were highly similar to that of previously sequenced beetles. Phylogenetic analyses based on three mitogenomic data and three analytical methods supported the monophyly of each of five superfamilies (Coccinelloidea, Chrysomeloidea, Curculionoidea, Tenebrionoidea and Cleroidea). The monophyly of Cucujoidea was never recovered in all the nine phylogenetic analyses. Tree topology tests statistically supported the superfamily-level relationships in Cucujiformia: ((Cleroidea+Coccinelloidea)+(Tenebrionoidea+(Cucujoidea (Chrysomeloidea+Curculionoidea)))). Although both mitogenomic datasets and analytical methods have an important impact on tree topologies, mitogenomic data have important implications in evolutionary and phylogenetic studies of Cucujiformia.

mitochondrial DNA, beetles, phylogenetic analysis, maximum likelihood method, Bayesian inference, topology test

doi: $10.1360 / \mathrm{N} 052018-00119$ 